\title{
The Effects of Ground and Treadmill Running on Energy Metabolism and Muscle Contractile Properties: A Randomized Cross-Over Study
}

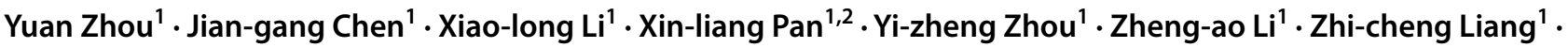 \\ Jia-min Long ${ }^{1} \cdot$ Ming-ze Qin ${ }^{1}$ · Jing Zhang ${ }^{1}$ (D) Bing Shi ${ }^{1} \cdot$ Bing-jun Wan ${ }^{1} \cdot$ Ai-ping Chi ${ }^{1} \cdot$ Wen-fei Zhu $^{1} \cdot \mathrm{Ke} \mathrm{Ning}^{1}$. \\ Yu-liang Sun ${ }^{1}$
}

Received: 25 February 2020 / Accepted: 24 August 2021 / Published online: 19 November 2021

(c) The Author(s) 2021

\begin{abstract}
Purpose This study aimed to compare the effects of ground and treadmill running on energy metabolism and muscle contractile properties, providing a basis for the general public to choose running venues.

Methods Ten male college students (age, $20.10 \pm 1.53$ years; height, $176.20 \pm 5.49 \mathrm{~cm}$; weight, $72.14 \pm 8.25 \mathrm{~kg}$; body fat percent, $12.41 \% \pm 4.65 \%$ ) were recruited in this study. Energy expenditure (EE) was measured using the accelerometer (GT9X) combined with specific estimation equations. Average heart rate (HR) was measured using a heart rate band (Polar). Muscle contractile properties were assessed by measuring muscle displacement $(\mathrm{Dm})$ and contraction velocity $(\mathrm{Vc}) \mathrm{using}$ tensiomyography (TMG-S1). Blood glucose (Glu) and lactate (Lac) were measured by portable devices (eB-G and Lactate Scout). The running speed was $9 \mathrm{~km} / \mathrm{h}$ and the duration was $25 \mathrm{~min}$. Two-way ANOVA (protocol $\times$ time) was used to analyze the effect of running protocols on energy metabolism and muscle contractile properties.

Results EE of treadmill running was significantly higher than EE of ground running (protocol main effect, $P<0.001$ ), and HR of treadmill running was significantly higher than that of ground running in the first testing time (protocol simple effect, $P=0.026$; protocol $\times$ time interaction $P=0.043$ ). The decrease in $\mathrm{Dm}$ of the rectus femoris after treadmill running was significantly higher than that of ground running (protocol main effect, $P=0.009$ ). The interaction of different running protocols and testing times on Lac was significant $(P=0.025)$, but all results of the simple effects analysis were not statistically significant $(P>0.05)$.

Conclusion Our study found a difference in energy expenditure between treadmill and ground running at $9 \mathrm{~km} / \mathrm{h}$ with duration of $25 \mathrm{~min}$. In addition, treadmills are more likely to cause a decrease in muscle displacement distance of the rectus femoris measured after exercise than ground running. Future studies are needed to further investigate whether the differences are induced by internal metabolism or the environmental conditions of running.
\end{abstract}

Keywords Ground running $\cdot$ Treadmill running $\cdot$ Energy expenditure $\cdot$ Muscle contractile properties $\cdot$ Blood glucose $\cdot$ Blood lactate

\section{Introduction}

Long-term regular exercises offer pronounced benefits to the body, and high levels of physical activity tend to be associated with a low risk of chronic diseases, as well as low mortality $[10,17]$. As a basic movement for people to carry out

Jing Zhang

zhangjiing0578@snnu.edu.cn

1 School of Physical Education, Shaanxi Normal University, No.620 West Chang'an Street, Chang' an District, Xi' an 710119, Shaanxi, China

2 School of Kinesiology, Beijing Sport University, No. 48 Xinxi Road, Haidian District, Beijing 100084, China their physical activities and varied sports, running has also been regarded as one of the most common and important methods of physical exercise.

The popular and public-accepted running methods can be roughly divided into two types: treadmill running and ground running. Ground running is one of the easiest and earliest forms of running, there is little additional equipment 
required to run on the ground. But ground running is affected by many environmental factors such as rain, snow, smog, muddy road, etc. Therefore, treadmill running, which is less affected by external environmental factors, is being chosen by more and more people for exercising indoors whenever. However, it is not clear whether treadmill running has the same effects on the body as ground running.

There is currently no consensus on how the two types of running affect the body differently. Li et al. [18] compared the difference between treadmill running and ground running in 74 middle-aged women and found that the energy expenditure of ground running was higher than treadmill running when the speed was at $8 \mathrm{~km} / \mathrm{h}$ and $9 \mathrm{~km} / \mathrm{h}$. Niemeyer et al. [24] came to a similar conclusion that the energy expenditure of interval training on an outdoor track was higher than that of incremental loading training with 30-s intervals on a treadmill. However, some studies have come to the opposite conclusion. A study with 13 high-level competitive athletes suggests that running in a natural environment in the wild may be more economical than running on a treadmill [23]. This discrepancy may be related to biomechanical adjustments. Several other studies have shown that energy expenditure may be higher when running on a treadmill [6, 30]. Differences in participants, running speeds and duration, and the order of the two running tests may also affect the final results.

The duration of the running session may be one of the first factors that need to be considered. Previous studies comparing the difference in energy expenditure between treadmill running and ground running have set multiple speed levels to test the difference in energy metabolism between the two types of running at different speeds $[1,18]$. But with such a design, the duration of each running speed is generally less than $10 \mathrm{~min}$. Participants may be forced to move to the next speed level before they can adapt, and this may inevitably affect energy expenditure. Second, the order of the two testing sessions may also have a potential effect on the outcome of the test. In some studies, the testing order of treadmill running and ground running was not random $[1,23]$, and the interval between two running tests was too short, which could affect the final test results. In addition, most studies did not include important indicators for monitoring aerobic exercise intensity such as blood glucose and blood lactate[15], as well as parameters for muscle contractile properties [22], which may reflect the state of the muscle or the fatigue level $[2,21]$. This makes it difficult to further compare the differences between the two running types.

Taken into account of the above factors, the purpose of this study was to investigate the effects of treadmill running and ground running with 25 min duration on energy expenditure, heart rate, blood glucose, blood lactate, and muscle contractile properties through a randomized cross-over design. We hypothesized that the effects of ground running and treadmill running on participants' energy expenditure, heart rate, blood glucose, blood lactate, and muscle contractile properties would be different. Theoretically, these results provide insights in selecting a more suitable sports venue for mass fitness crowd or competitive professional training.

\section{Methods}

\section{Participants}

Ten male college students with similar running habits, with an average age of $20.10 \pm 1.53$ years were selected as participants. A bicycle ergometer (ergoselect 100, Bitz, Germany) was used to assess the endurance ability of participants. Participants were asked to pedal continuously for $6 \mathrm{~min}$ at a load of $100 \mathrm{w}$. The average heart rate at the 5 th and 6 th minutes was calculated and plotted on an Astrand-Ryhming nomogram to obtain the indirect maximal oxygen uptake. A smartphone app (Keep) was used to record the participants' running distance for about a month. The characteristics of participants are presented in Table 1. All participants were asked to wear shoes with the same characteristics, that is, no more than 4 to $6 \mathrm{~mm}$ between the front and back of the shoe, and the midsole was made of ethylene-vinyl acetate copolymer. Participants were asked to refrain from vigorous activity 3 days before the first test and between the first and second tests. Each test was conducted $3 \mathrm{~h}$ after the participants had food. Participants were informed of the possible risks before the test and signed the informed consent forms. The protocols were approved by the Ethics Committee of the University. The ethical approval number is 202016004.

\section{Study Design}

This study is a randomized cross-over study. Participants were randomly assigned to group A and group B by a computer program (Fig. 1). In group A, treadmill running was performed first and proceeded with ground running afterward. For group B, ground running was performed first and

Table 1 Characteristics of participants (mean \pm SD) $n=10$

\begin{tabular}{lc}
\hline Age $($ years $)$ & $20.10 \pm 1.53$ \\
Height $(\mathrm{cm})$ & $176.20 \pm 5.49$ \\
Weight $(\mathrm{kg})$ & $72.14 \pm 8.25$ \\
Body mass index $\left(\mathrm{kg} / \mathrm{m}^{2}\right)$ & $23.16 \pm 5.31$ \\
Skeletal muscle mass index $\left(\mathrm{kg} / \mathrm{m}^{2}\right)$ & $12.02 \pm 1.34$ \\
Body fat percent $(\%)$ & $12.41 \pm 4.65$ \\
Vital capacity $(\mathrm{mL})$ & $4800.42 \pm 863.97$ \\
$\mathrm{VO}_{2 \max }(\mathrm{mL} / \mathrm{kg} / \mathrm{min})$ & $36.83 \pm 7.38$ \\
Weekly running distance $(\mathrm{km})$ & $10.23 \pm 2.47$ \\
\hline
\end{tabular}



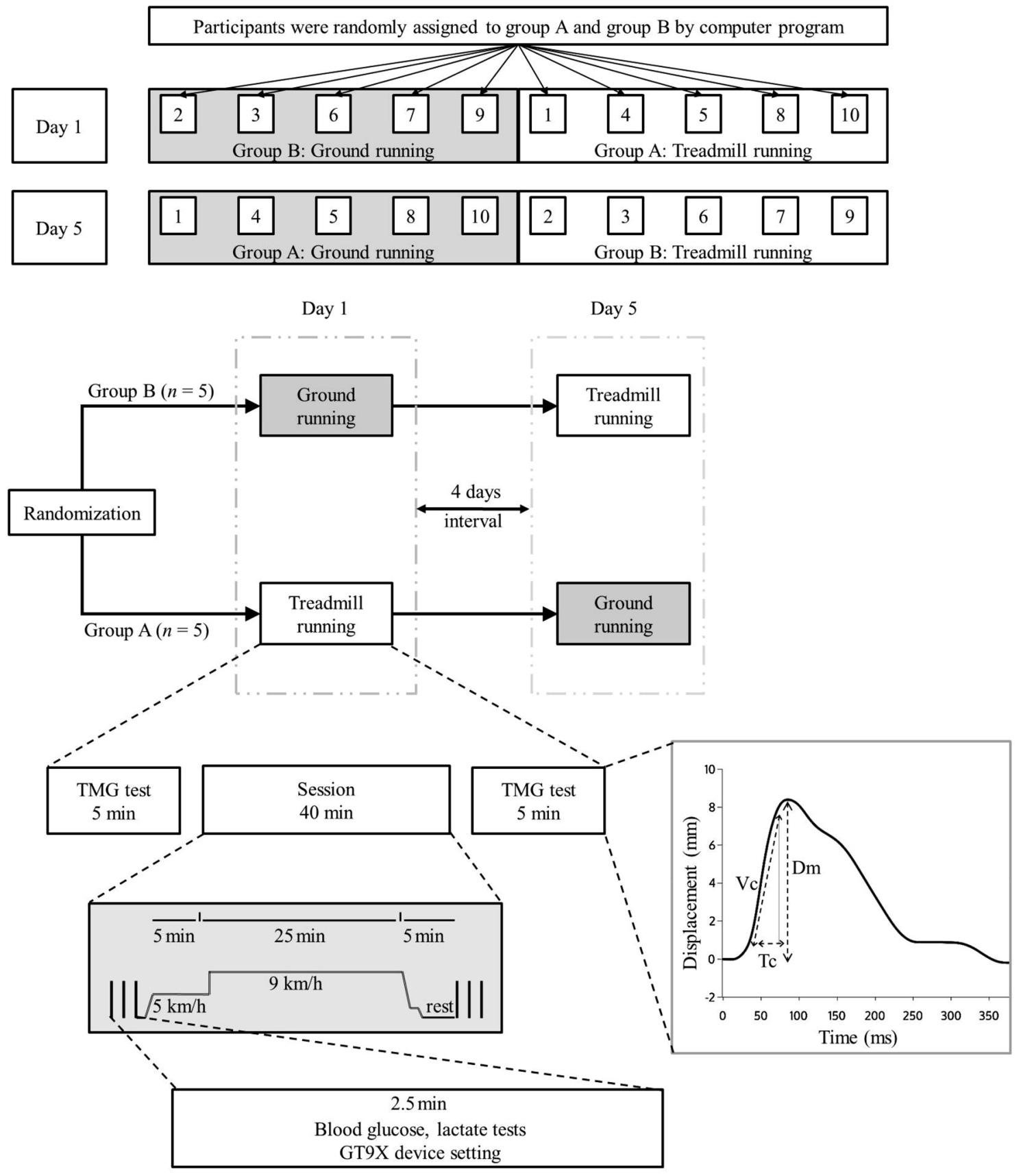

Fig. 1 Study design. $T M G$ tensiomyography; $D m$ displacement; $T c$ contraction time. $V c$ contraction velocity, $[\mathrm{Vc}=(90 \% \mathrm{DM}-10 \% \mathrm{DM}) / \mathrm{Tc}]$

proceeded with treadmill running afterward. A 4-day interval was set between the treadmill running and the ground running to eliminate the effects of fatigue.

\section{Procedures}

The treadmill running tests were completed in an indoor laboratory. Air conditioning was used to adjust the lab temperature to a similar 16 degrees Celsius to the outdoor temperature. After warming up for 5 min with a speed of $5 \mathrm{~km} / \mathrm{h}$ on the treadmill, the participants were informed to run at a speed of $9 \mathrm{~km} / \mathrm{h}$ for $25 \mathrm{~min}$. The treadmill model used was quasar $^{\circledR}$ (h/p/cosmos, Munich, Germany) and was equipped with a heart rate band (Polar Electro, Finland) to detect the real-time heart rate.

The ground running tests were performed at an outdoor gymnasium $200 \mathrm{~m}$ from the laboratory. The participants performed the same exercise on the outdoor ground under the guidance of professional personnel. After warming up at approximately $5 \mathrm{~km} / \mathrm{h}$ for $5 \mathrm{~min}$, participants keep running 
at $9 \mathrm{~km} / \mathrm{h}$ for $25 \mathrm{~min}$. The outdoor running ground was a 400-m circle and was flat without any slopes. Disc cones (SC01, Teamforce, Shanghai) were placed every $10 \mathrm{~m}$ as markers. A professional metronome smartphone app (Jie Pai Qi, Wuhan, China) was used to help participants adjust their speed to pass a disc cone every four sound signals. The duration of every four sound signals was $4 \mathrm{~s}$. The sound signal is a counting cycle of the human voice from 1 to 4 . The heart rate was monitored using a polar heart rate band connected to the GT9X (ActiGraph, Pensacola, USA).

Running at a speed of $9 \mathrm{~km} / \mathrm{h}$ is classified as high-intensity exercise [25]. Also, 25-30 min of high-intensity exercise per day produces health benefits [11]. By considering the tolerable amount of daily physical activity intensity of the human body and from a health perspective, this study was set at a speed of $9 \mathrm{~km} / \mathrm{h}$ running for $25 \mathrm{~min}$.

\section{Anthropometric Measurements}

Skeletal muscle mass and body fat percent were measured by a body composition analyzer (Inbody 230, Biospace, Korea). Height and weight were measured by an all-in-one machine (GK 720, Shandong, China), which integrates an electronic weight scale and an ultrasonic stadiometer. Body mass index (BMI) was automatically calculated by GK720. The skeletal muscle mass index is obtained by dividing the skeletal muscle mass by the square of the height.

\section{Energy-Expenditure Tests}

The energy expenditure (EE) was measured using GT9X. The GT9X is positioned at the right ankle and is wirelessly connected to the heart rate band (Polar Electro, Finland) for real-time heart rate monitoring. The EE estimation equation was adjusted using heart rate reserve (HRR) and body weight (BW). Direct or indirect calorimetry and double-standard water method assist in accurately calculating the energy expenditure in motion, but their requirements are strict and restrictive. Therefore, the accelerometer is taken as an objective measurement method due to its high reliability and easy operation and is more and more widely used. The estimation equation of energy expenditure was developed by Ho et al. [13], which has been adapted specifically for non-athletes with exercise habits. This equation shows good reliability and validity $(r=0.946, I C C=0.945)$. The equation is as follows:
ActliLife6 (Version 6.13.4, ActiGraph, USA) software. The software provides multiple 10-s lengths of vector magnitude sums, adding up 15010 -s vector magnitude sums to get the total vector magnitude.

\section{Blood Glucose and Lactate Tests}

The blood glucose levels were measured by eBsensor Blood Glucose Monitoring System (eB-G). The eB-G (VISGENEER Inc, Taiwan, China) includes comfortable blood glucose testing equipment and endurance comfortable test paper. The blood lactate levels were measured by lactate scout portable lactate analyzer (senslab GmbH, Germany) and LactateScout Sensorstest paper (senslab GmbH, Germany). Both blood glucose and lactate were measured in participants at rest and $5 \mathrm{~min}$ after finishing the running session. All biochemical indexes were tested by fingertip blood sampling. The devices were calibrated strictly according to the instructions before the experiment and the participants were asked to have the same diet before the running session to minimize the variabilities during the experimental process.

\section{Tensiomyography Tests}

The tensiomyography (TMG) was assessed using TMG S1(TMG-BMC, Ljubljana, Slovenia), which combined a highly sensitive displacement transducer with an electrical stimulator. TMG has served as a simple and non-invasive tool to assess the mechanical properties of skeletal muscles [19]. It can be carried out quickly, without producing additional fatigue, and does not depend on voluntary motivation [26]. Several contractile parameters can be derived from this technique, such as maximal muscle displacement (DM), contraction time (Tc), delay time (Td), and the TMG-derived velocity of contraction $(\mathrm{Vc})$ [19]. Vc is calculated from the ratio between $10 \%$ to $90 \%$ of DM and $\mathrm{Tc}$ [21]. These indicators can be especially useful to assess the rigidity, mechanical characteristics, and contractile capacity of superficial muscles when activated by an electrical stimulus of controlled intensity [29]. In our experiment, two electrodes were attached to the thickest part of the rectus femoris muscle of the right leg and the digital displacement transducer was placed vertically. An initial current amplitude of $20 \mathrm{~mA}$ was applied. This amplitude was gradually increased at a rate of $10 \mathrm{~mA}$ until the maxi-

$\mathrm{GT} 9 \mathrm{X}-\mathrm{EE}(\mathrm{kcal} / \mathrm{min})=0.000004 \times \mathrm{VM}-0.000417 \times \mathrm{BW}-0.000478 \times \mathrm{HRR}+0.031277$,

$3 \mathrm{D} \mathrm{VM}=\sqrt{(\operatorname{axis} 1)^{2}+(\operatorname{axis} 2)^{2}+(\operatorname{axis} 3)^{2}}$.

The three-dimensional (3D) vector magnitude (VM, triaxial accelerometer data combination) was derived from the mum response was reached. The end of the test was marked consistently when repositioning the sensor for subsequent measurements [20]. Previous studies have suggested that contractile properties such as muscle displacement distance 
and contraction velocity can reflect muscle fatigue to some extent [14, 21, 27].

\section{Statistical Analysis}

Shapiro-Wilk test was used to test the normal distribution of data. Descriptive statistics were expressed as means \pm standard deviation (SD). Two-way ANOVA $(2 \times 2)$ was used to analyze the effect of running protocols (ground running vs. treadmill running) and the two running times (first run vs. second run) on VM, EE, HR, Glu, Lac, Vc, and Dm. Levene's test showed equal variance and box plot test showed no outliers. Main effects analysis was used when the interaction effect was not significant, and simple effects analysis was used the interaction effect was significant. $P<0.05$ was considered as significant level, and $P<0.01$ was considered as very significant level. SPSS 25.0 software was used to analyze the data.

\section{Results}

The effects of different running protocols on energy expenditure and heart rate are shown in Table 2. There was no interaction between the effects of the different protocols and testing times on the energy expenditure $(P=0.394)$. The results of the main effects analysis showed that the effect of running protocol on energy expenditure was statistically significant, and the energy expenditure of treadmill running was higher than that of ground running $(P<0.001)$ (Fig. 2A). The effect of different running protocols and testing times on average heart rate had an interactive effect $(P=0.043)$. The simple effects analysis showed that the average heart rate of treadmill running was significantly higher than that of ground running in the first test $(\mathrm{GO}, 148.00 \pm 13.64 \mathrm{vs}$. TM, $164.60 \pm 11.15$ beats $/ \mathrm{min}, P=0.026$ ).

The effects of different running protocols on glucose, lactate, contraction velocity, and displacement distance of the rectus femoris are shown in Table 3. There was no interaction between the effects of different running protocols and testing times on glucose, muscle contraction velocity, or muscle displacement distance $(P>0.05)$. The main effect analysis showed that the effect of running protocol on muscle displacement distance was statistically significant, and the decrease in muscle displacement distance of treadmill running was significantly higher than that of ground running $(P=0.009)$ (Fig. 3D). There was an interaction between the effects of different running protocols and testing times on lactate $(P=0.025)$ (Table 3$)$. But the results of the simple effects analysis showed that neither the running protocol nor the testing time was statistically significant $(P>0.05)$.
Table 2 Energy expenditure and average heart rate during ground and treadmill running $($ mean $\pm \mathrm{SD})$

\begin{tabular}{|c|c|c|c|c|c|}
\hline \multirow[t]{2}{*}{ Variable } & \multicolumn{2}{|l|}{$9 \mathrm{~km} / \mathrm{h}$} & \multicolumn{3}{|l|}{$P$ value } \\
\hline & GO & $\mathrm{TM}$ & Protocol & Time & protocol $\times$ time \\
\hline $\mathrm{EE}(\mathrm{kcal} / \mathrm{kg} / \mathrm{min})$ & $0.16 \pm 0.01$ & $0.17 \pm 0.01$ & $<0.001$ & 0.612 & 0.394 \\
\hline HR (beats/min) & $153.10 \pm 10.75$ & $159.20 \pm 12.15$ & 0.219 & 0.951 & 0.043 \\
\hline
\end{tabular}

$G O$ ground running; $T M$ treadmill running; $E E$ energy expenditure; $H R$ average heart rate

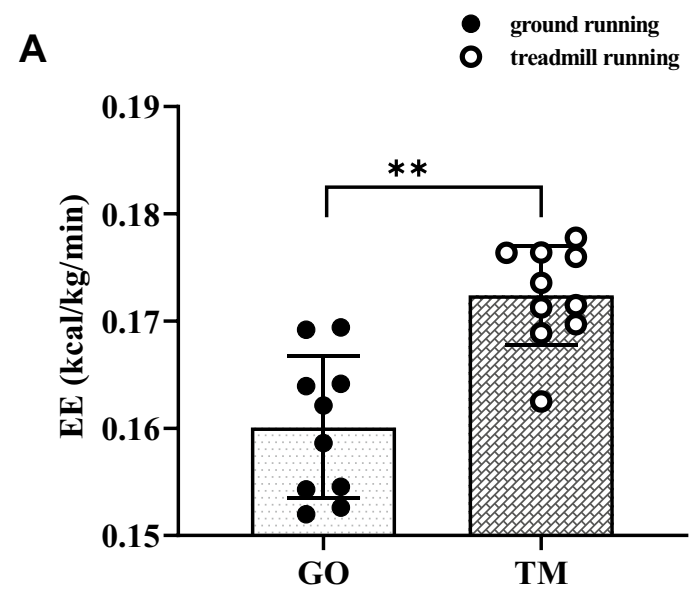

Fig. 2 A Comparison of the energy expenditure (EE) between ground running and treadmill running. B Comparison of the average heart rate between ground running and treadmill running. Data are repre-
B

ground running

treadmill running

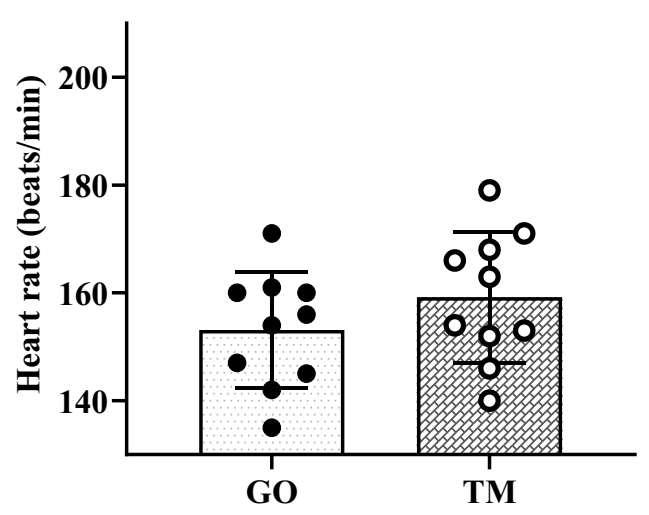

sented by mean $\pm \mathrm{SD}$; $G O$ ground running; $T M$ treadmill running; ** indicates significant difference between ground running and treadmill running, $P<0.01$ 
Table 3 Change of blood glucose, blood lactate, muscle contraction velocity, and muscle displacement after ground and treadmill running $($ mean $\pm \mathrm{SD})$

\begin{tabular}{|c|c|c|c|c|c|c|}
\hline \multirow[t]{2}{*}{ Variable } & \multicolumn{3}{|l|}{$9 \mathrm{~km} / \mathrm{h}$} & \multicolumn{3}{|l|}{$P$ value } \\
\hline & Pre & Post & Change & Protocol & Time & Protocol $\times$ time \\
\hline \multicolumn{7}{|c|}{ Glu (mmol/L) } \\
\hline GO & $4.64 \pm 0.99$ & $4.30 \pm 0.27$ & $-0.34 \pm 1.38$ & 0.781 & 0.960 & 0.149 \\
\hline $\mathrm{TM}$ & $4.33 \pm 0.80$ & $4.19 \pm 1.42$ & $-0.14 \pm 1.71$ & & & \\
\hline \multicolumn{7}{|c|}{$\mathrm{Lac}(\mathrm{mmol} / \mathrm{L})$} \\
\hline GO & $1.65 \pm 0.80$ & $1.91 \pm 0.64$ & $0.27 \pm 0.68$ & 0.898 & 0.655 & 0.025 \\
\hline TM & $1.83 \pm 0.82$ & $2.06 \pm 0.35$ & $0.23 \pm 0.85$ & & & \\
\hline \multicolumn{7}{|c|}{$\mathrm{Vc}(\mathrm{mm} / \mathrm{ms})$} \\
\hline GO & $0.18 \pm 0.06$ & $0.16 \pm 0.04$ & $-0.02 \pm 0.02$ & 0.838 & 0.641 & 0.860 \\
\hline $\mathrm{TM}$ & $0.15 \pm 0.04$ & $0.13 \pm 0.04$ & $-0.02 \pm 0.04$ & & & \\
\hline \multicolumn{7}{|c|}{$\mathrm{Dm}(\mathrm{mm})$} \\
\hline GO & $6.01 \pm 1.98$ & $5.79 \pm 1.82$ & $-0.22 \pm 0.69$ & 0.009 & 0.651 & 0.556 \\
\hline $\mathrm{TM}$ & $5.89 \pm 1.81$ & $4.60 \pm 1.53$ & $-1.28 \pm 0.84$ & & & \\
\hline
\end{tabular}

$G O$ ground running; $T M$ treadmill running; $G l u$ blood glucose; Lac blood lactate; $V c$ contraction velocity of rectus femoris; $D m$ displacement of rectus femoris

\section{Discussion}

This study aimed to compare the effects of ground and treadmill running on energy expenditure, heart rate, blood glucose, blood lactate, and muscle contractile properties. The main findings of this study were that the energy expenditure during treadmill running was significantly higher than that during ground running and that the average heart rate of treadmill running was significantly higher than that of ground running in the first testing session. We also observed that displacement of the rectus femoris muscle decreased more after treadmill running than ground running. There was no significant difference in blood glucose or blood lactate after ground and treadmill running.

\section{Effect of Ground and Treadmill Running on Energy Expenditure and Possible Mechanisms}

The results of this study showed that the energy expenditure during treadmill running was higher than that during ground running. Edwards et al. [6] also found that both treadmill running and non-motorized treadmill running showed higher oxygen consumption and perceived exertion than ground running at speed of $10.5 \mathrm{~km} / \mathrm{h}$. In addition to running, walking on a treadmill also appeared to show higher energy expenditure than walking on the ground. Berryman et al. [1] compared the energy expenditure of walking on the ground with that of walking on a treadmill in older adults and found that the treadmill required more energy at walking speeds of $2.4-5.6 \mathrm{~km} / \mathrm{h}$. In a study with young people, Dal et al. [3] compared the speed of self-selection and energy expenditure of walking on a treadmill and the ground. The authors found that the self-selected speed of treadmill walking was significantly lower than that of ground walking. Furthermore, the oxygen consumption on the treadmill at the preferred speed of the treadmill was significantly greater than that on the ground at the preferred speed of the ground.

The mechanism for the difference in energy expenditure between ground and treadmill running is not yet clear. One possible explanation is that treadmill running requires more balance and coordination than ground running [3]. Another possible explanation is the difference in hardness between the surface of the ground and the treadmill. During running, the reaction force of the ground pushes the human body forward. However, the shock absorption of the treadmill reduces the reaction force of its surface on the human body. Therefore, to meet the requirements of the set running speed, the body increases its force to backpedal the ground to meet the needs of exercise, which may increase energy expenditure. Hardin et al. [12] have found that with increased ground hardness, the flexion angle of the hip and knee was decreased when contacting the ground, and the maximum flexion angle of the hip was decreased. During running on the ground with low hardness, the flexion angle and the maximum flexion angle of the hip and knee joint in the first touchdown period and mid-support period were larger than those on the hard ground [28]. Because of the shock absorber system of the treadmill, the flexion angle of the hip and knee joint of the body was larger than that on the ground during treadmill running. Therefore, more energy was consumed by the body to complete a movement. With increased flexion angle of the hip and knee joints when running on the treadmill, more work was done on the treadmill per unit time, and more energy was consumed.

\section{Effect of Ground and Treadmill Running on Muscle Contractile Properties}

Our analysis of the change in the rectus femoris muscle after ground and treadmill running revealed that the decrease in 
A

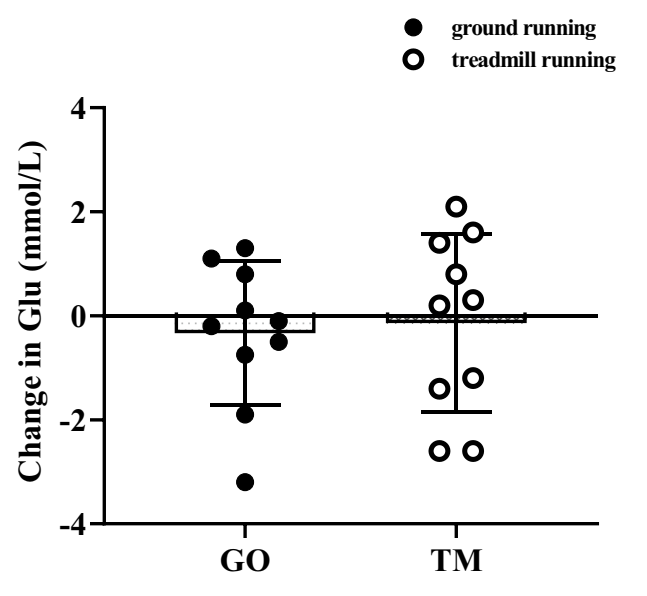

C

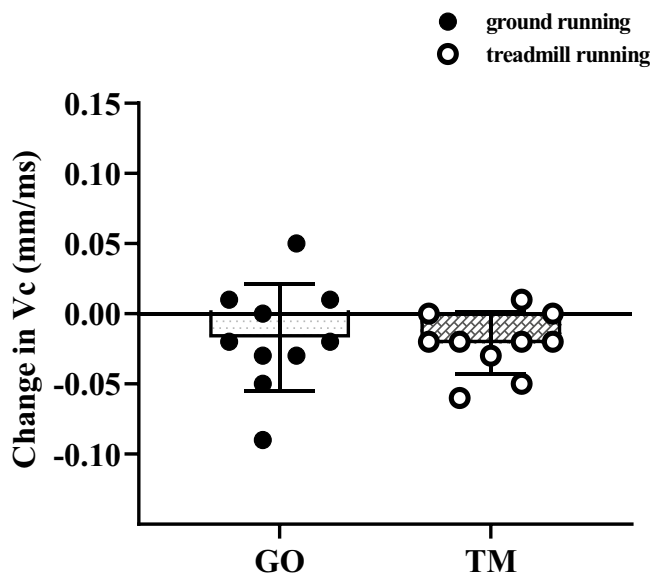

Fig. 3 A Comparison of the change in blood glucose (Glu) between ground and treadmill running. B Comparison of the change in blood lactate (Lac) between ground and treadmill running. C Comparison of the change in contraction velocity $(\mathrm{Vc})$ of rectus femoris between ground and treadmill running. D Comparison of the change in dis-

maximum displacement of rectus femoris muscle after treadmill running was significantly higher than that of ground running. Muscle displacement, as a valid indicator for evaluating muscle fatigue, has been reported in an increasing number of studies [7, 20]. One previous study using TMG to assess exercise-induced muscle injury observed that the decline in Dm followed the same profile as the decline in muscle force. Further, the authors found a strong relationship between muscle displacement and creatine kinase levels and muscle soreness [14]. Another study found that different types of lower extremity resistant training compared to baseline measurements all resulted in reduced muscle displacement in 14 athletes [27]. The authors believe that TMG muscle contractile properties are sensitive to changes in muscle force, and the more repetitive the exercise, the more likely it is to cause changes in TMG muscle characteristics. In this study, all participants seemed to experience a decrease in muscle displacement after running on a treadmill, while a
B

- ground running

- treadmill running

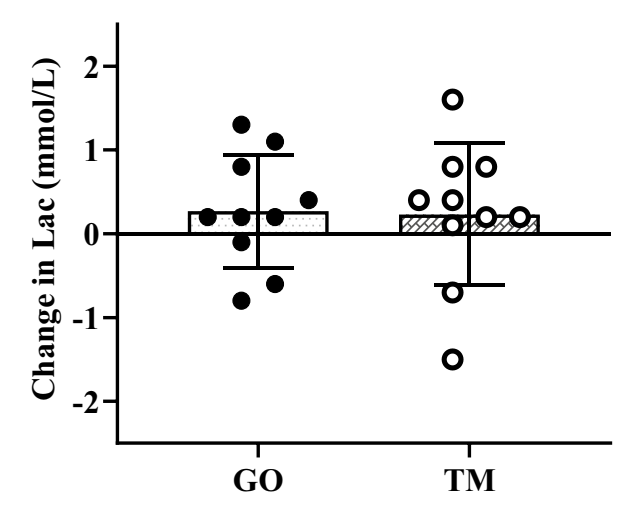

D

ground running

- treadmill running

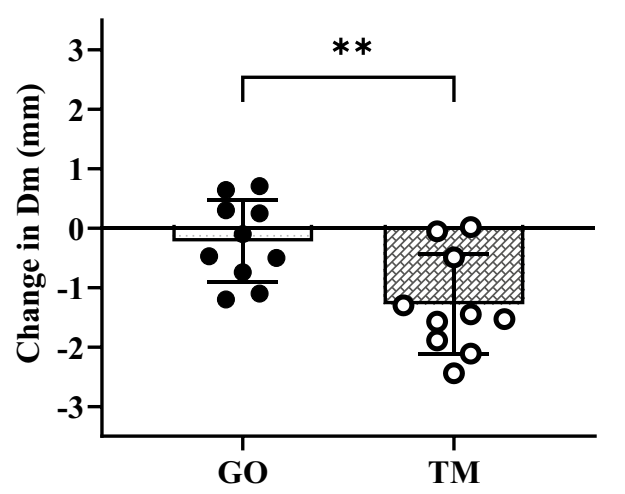

placement (Dm) of rectus femoris between ground and treadmill running. Data are represented by mean $\pm \mathrm{SD}$; GO: ground running; TM: treadmill running; $* *$ indicates a significant difference between ground and treadmill running, $P<0.01$

few participants seemed to experience an increase in muscle displacement after ground running. This may indicate that the muscles were more fatigued after treadmill running than ground running, probably because the treadmill environment is more restricted than the ground environment, and therefore body may need more neuromuscular control to maintain coordination and balance. Studies involving biomechanical indicators such as EMG and gait parameters appear to support this view. Mileti et al. [22] measured the EMG of lower limbs while running on a treadmill at a speed of $9 \mathrm{~km} / \mathrm{h}$ and found that the activation pattern of muscles during treadmill exercise was more regular and constrained. This regular neural control strategy may stem from the sensory constraints imposed by the treadmill environment, and the authors suggested that treadmills might have a considerable impact on motor coordination. Another explanation involves differences in the mechanics of running in lower extremity muscles due to differences between the ground 
and the treadmill surface [4]. Based on multivariate information fusion algorithm, comparative analysis of the data of knee joint rotation angle and gait phase of 20 healthy young men walking on flat and treadmill, Zhao et al. [31] revealed that treadmill walking was less stable than walking on a flat surface, and the range of knee joint rotation angle was 2-3 times more than that walking on the flat. The moment the heel touches the ground during walking on the treadmill, the range of knee joint rotation angle was obviously bigger than that on the flat. Namely, the heel touches the ground when the calf cannot be completely maintained straightly. To ensure the balance and stability of walking, people subjectively make the swing legs fall on the ground ahead of time to shorten the swing time and increase the contact time of both feet on the treadmill at the same time. This may explain why treadmill running and ground running have different effects on muscle contractile properties.

\section{Effect of Ground and Treadmill Running on Blood Glucose and Lactate}

Blood glucose concentration refers to glucose content per liter of blood, and the blood glucose concentration usually remains within a narrow range $(3.5-6.5 \mathrm{mmol} / \mathrm{L})$ [5]. By regulating the circulatory substrates and reducing the activity of fructokinase phosphate, exercise can dramatically reduce the utilization rate of glucose during the glycolysis process in maintaining normal blood glucose levels [9]. The results of this study showed that after the ground and treadmill running for $25 \mathrm{~min}$, almost no difference was observed in the change of blood glucose concentration, suggesting that after 25 min of running on the ground and treadmill at the same speed, the body consumed the same amount of sugar. The results in this study also showed no difference in the change of blood lactate concentration after ground and treadmill running for $25 \mathrm{~min}$. This may indicate that the anaerobic metabolic energy supply ratio during treadmill running was the same as that during ground running. Blood lactate concentration acts as a biochemical index that is commonly used in sports training, which can scientifically evaluate the intensity of the exercise load [16]. In this study, participants' lactate did not change much regardless of the type of running protocol, which may be due to the better aerobic endurance level of the participants and the body's predominant use of aerobic energy supply. Blood lactate depends on the balance between lactate production rate $(\mathrm{RaL})$ and lactate depletion rate (RdL). During exercise, RaL depends on the lactate produced in contracting muscles, while RdL depends on gluconeogenesis or the rate of lactate uptake by cell respiration in tissues. We even observed a decrease in lactate in some participants, which may be the result of the body gradually overcoming visceral inertia and metabolizing the lactate produced. On the other hand, when exercise load was increased, RaL exceeds RdL, and blood lactate level showed a continuous increase [8]. Therefore, this may also indicate that the participants adapted to the intensity of the exercise. Our study found an interaction effect between running protocol and test time, but none of the simple effects analyses were significant. This may need to be clarified in future studies with larger samples.

One of the strengths of this study is that we explored the differences in blood glucose, blood lactate, and muscle fatigue between ground running and treadmill running for the first time, providing new insights into the choice of running venues. Secondly, this study is a randomized cross-over design, which can effectively eliminate the potential impact of ground running and treadmill running test sequences. However, it should be mentioned that this study also has some limitations. Firstly, the participants recruited in this study are mainly young people around the age of 20 , who have good sports ability. Therefore, the results of this study are not suitable to be generalized to all ages. Secondly, due to the strict inclusion criteria and other factors, the sample size of this study is relatively small. The sample size in future studies should be increased. Finally, this study did not measure biomechanical parameters such as EMG and joint motion angle. These indicators can provide more information for the effects of ground and treadmill running on energy metabolism and muscle fatigue and should be included in future research.

\section{Conclusions}

Our study found a difference in energy expenditure between treadmill and ground running at $9 \mathrm{~km} / \mathrm{h}$ for $25 \mathrm{~min}$. However, it needs to be studied whether this phenomenon is caused by the internal metabolism or the environmental conditions of running. In addition, the two running protocols have different effects on the contractile properties of the muscles. After treadmill running, the displacement distance of the rectus femoris muscle decreases significantly more than that of the ground running.

Acknowledgements The authors would like to thank all the participants and experimenters for their efforts in the experiment process and their serious and responsible attitude is commendable.

Author contributions JZ designed the study, supervised the process of experiment and data collection, and reviewed and revised the manuscript. JC, YZ, BS, BW, AC, WZ, KN, and YS recruited the participants and assisted the data collection, contributed to the statistical analyses, and initial drafting of the manuscript. JC, YZ, XL, XP, YZ, $\mathrm{ZL}$, and $\mathrm{LZ}$ contributed to the data interpretation and reviewed and revised the manuscript. JC and JL prepared figures in the manuscript. 
All authors approved the final manuscript as submitted and agreed to be accountable for all aspects of the work.

Funding No funding.

Availability of Data and Material Not applicable.

Code Availability Not applicable.

\section{Declarations}

Conflict of interest We declare no competing interests.

Ethical approval The protocols were approved by the Ethics Committee of a University.

Open Access This article is licensed under a Creative Commons Attribution 4.0 International License, which permits use, sharing, adaptation, distribution and reproduction in any medium or format, as long as you give appropriate credit to the original author(s) and the source, provide a link to the Creative Commons licence, and indicate if changes were made. The images or other third party material in this article are included in the article's Creative Commons licence, unless indicated otherwise in a credit line to the material. If material is not included in the article's Creative Commons licence and your intended use is not permitted by statutory regulation or exceeds the permitted use, you will need to obtain permission directly from the copyright holder. To view a copy of this licence, visit http://creativecommons.org/licenses/by/4.0/.

\section{References}

1. Berryman N, Gayda M, Nigam A, Juneau M, Bherer L, Bosquet L. Comparison of the metabolic energy cost of overground and treadmill walking in older adults. Eur J Appl Physiol. 2012;112(5):1613-20.

2. Ce E, Longo S, Limonta E, Coratella G, Rampichini S, Esposito F. Peripheral fatigue: new mechanistic insights from recent technologies. Eur J Appl Physiol. 2020;120(1):17-39.

3. Dal U, Erdogan T, Resitoglu B, Beydagi H. Determination of preferred walking speed on treadmill may lead to high oxygen cost on treadmill walking. Gait Posture. 2010;31(3):366-9.

4. Dingwell JB, Cusumano JP, Cavanagh PR, Sternad D. Local dynamic stability versus kinematic variability of continuous overground and treadmill walking. J Biomech Eng. 2001;123(1):27-32.

5. Dunning T. How to monitor blood glucose. Nurs Stand. 2016;30(22):36-9.

6. Edwards RB, Tofari PJ, Cormack SJ, Whyte DG. Non-motorized treadmill running is associated with higher cardiometabolic demands compared with overground and motorized treadmill running. Front Physiol. 2017;8:914.

7. García-Manso JM, Rodríguez-Matoso D, Sarmiento S, Saa YD, Vaamonde D, Rodríguez-Ruiz D, Silva-Grigoletto MED. Effect of high-load and high-volume resistance exercise on the tensiomyographic twitch response of biceps brachii. J Electromyogr Kinesiol. 2012;22(4):612-9.

8. García-Suárez PC, Rentería I, Moncada-Jiménez J, Fry AC, Jiménez-Maldonado A. Acute systemic response of BDNF, lactate and cortisol to strenuous exercise modalities in healthy untrained women. Dose-Response. 2020;18(4):500551495.
9. Gibb AA, Epstein PN, Uchida S, Zheng Y, Mcnally LA, Obal D, Katragadda K, Trainor PJ, Conklin DJ, Brittian KR. Exerciseinduced changes in glucose metabolism promote physiological cardiac growth. Circulation. 2017;136(22):2144-57.

10. Gilders RM, Voner C, Dudley GA. Endurance training and blood pressure in normotensive and hypertensive adults. Med Sci Sports Exerc. 1989;21(6):629-36.

11. Giroir BP, Wright D. Physical activity guidelines for health and prosperity in the United States. JAMA. 2018;320(19):1971-2.

12. Hardin EC, van den Bogert AJ, Hamill J. Kinematic adaptations during running: effects of footwear, surface, and duration. Med Sci Sports Exerc. 2004;36(5):838-44.

13. Ho CS, Chang CH, Hsu YJ, Tu YT, Huang CC. Feasibility of the energy expenditure prediction for athletes and non-athletes from ankle-mounted accelerometer and heart rate monitor. Sci Rep. 2020;10(1):8816.

14. Hunter AM, Galloway SD, Smith IJ, Tallent J, Ditroilo M, Fairweather MM, Howatson G. Assessment of eccentric exerciseinduced muscle damage of the elbow flexors by tensiomyography. J Electromyogr Kinesiol. 2012;22(3):334-41.

15. Junior PB, Andrade VLD, Campos EZ, Kalva-Filho CA, Zagatto AM, Araújo GGD, Marcelo P. Effect of endurance training on the lactate and glucose minimum intensities. J Sports Sci Med. 2018;17(1):117-23.

16. Karlsen T, Solli GS, Samdal ST, Sandbakk O. Intensity control during block-periodized high-intensity training: heart rate and lactate concentration during three annual seasons in world-class cross-country skiers. Front Sports Act Living. 2020;2:549407.

17. Kokkinos P, Myers J. Exercise and physical activity: clinical outcomes and applications. Circulation. 2010;122(16):1637-48.

18. Li S, Xue JJ, Hong P, Song C, He ZH. Comparison of energy expenditure and substrate metabolism during overground and motorized treadmill running in Chinese middle-aged women. Sci Rep. 2020;10(1):1815.

19. Loturco I, Pereira LA, Kobal R, Kitamura K, Ramirez-Campillo $\mathrm{R}$, Zanetti V, et al. Muscle contraction velocity: a suitable approach to analyze the functional adaptations in elite soccer players. J Sports Sci Med. 2016;15(3):483-91.

20. Macgregor LJ, Ditroilo M, Smith IJ, Fairweather MM, Hunter AM. Reduced radial displacement of the gastrocnemius medialis muscle after electrically elicited fatigue. J Sport Rehabil. 2016;25(3):241-7.

21. Macgregor LJ, Hunter AM, Orizio C, Fairweather MM, Ditroilo M. Assessment of skeletal muscle contractile properties by radial displacement: the case for tensiomyography. Sports Med. 2018;48(7):1607-20.

22. Mileti I, Serra A, Wolf N, Munoz-Martel V, Santuz A. Muscle activation patterns are more constrained and regular in treadmill than in overground human locomotion. Front Bioeng Biotechnol. 2020;8:581619.

23. Mooses M, Tippi B, Mooses K, Durussel J, Maestu J. Better economy in field running than on the treadmill: evidence from high-level distance runners. Biol Sport. 2015;32(2):155-9.

24. Niemeyer M, Weber T, Beneke R. Higher energy and carbohydrate demand of interval training at a given average velocity on track versus treadmill. Appl Physiol Nutr Metab. 2019;44(4):447-9.

25. Norton K, Norton L, Sadgrove D. Position statement on physical activity and exercise intensity terminology. J Sci Med Sport. 2010;13(5):496-502.

26. Simola RADP, Harms N, Raeder C, Kellmann M, Ferrauti A. Assessment of neuromuscular function after different strength training protocols using tensiomyography. J Strength Cond Res. 2015;29(5):1339-48.

27. de Paula SR, Raeder C, Wiewelhove T, Kellmann M, Meyer T, Pfeiffer M, Ferrauti A. Muscle mechanical properties of strength 
and endurance athletes and changes after one week of intensive training. J Electromyogr Kinesiol. 2016;30:73-80.

28. Pinnington HC, Lloyd DG, Besier TF, Dawson B. Kinematic and electromyography analysis of submaximal differences running on a firm surface compared with soft, dry sand. Eur J Appl Physiol. 2005;94(3):242-53.

29. Rodriguez-Ruiz D, Diez-Vega I, Rodriguez-Matoso D, Fernandez-del-Valle M, Sagastume R, Molina JJ. Analysis of the response speed of musculature of the knee in professional male and female volleyball players. Biomed Res Int. 2014;2014:239708.

30. Yngve A, Nilsson A, Sjostrom M, Ekelund U. Effect of monitor placement and of activity setting on the MTI accelerometer output. Med Sci Sports Exerc. 2003;35(2):320-6.

31. Zhao L, Huang S, Sui L, Li J. Differences between knee movements walking on flat ground and on a treadmill. J Harbin Eng Univ. 2015;36(9):1259-63. 